\title{
Der Gartenarchitekt Henry Kraft und der Görlitzer Berggarten
}

\author{
Christoph Haase
}

$\mathrm{M}$ it ihren vielen Gärten, Parks, Plätzen, Friedhöfen und bepflanzten Straßenzügen ist Görlitz eine bemerkenswert grüne Stadt. Nicht umsonst hatte sie bereits im 19. Jahrhundert den Ruf einer Gartenstadt und war unter anderem beliebter Alterssitz wohlhabender Pensionäre. Mit Beginn der 1830er Jahre setzte eine wahre Welle der Verschönerung öffentlicher wie privater Freiräume in der wirtschaftlich aufstrebenden Stadt ein. Eine seinerzeit eigens ins Leben gerufene Verschönerungs-Deputation sorgte für die Anlegung und Pflege von Parks und Promenaden. Bis zum Beginn des 20. Jahrhunderts entwickelte sich sukzessive ein weitläufiges Grünsystem, dessen Herzstück von Anfang an der Stadtpark darstellte. Auch die um die Jahrhundertwende zahlreich entstandenen Villengärten trugen zu einer Aufwertung des Stadtbildes bei. ${ }^{1}$

Wie anderen Orts stellte der Zweite Weltkrieg eine harte Zäsur dar und bewirkte zunächst einen Stillstand in der Entwicklung des Stadtgrüns. In den 1950er und 1960er Jahren kam es jedoch zu einer neuen Blütezeit, was maßgeblich dem geschickten und verdienstvollen Wirken des Görlitzer Gartenbaudirektors Henry Kraft zu verdanken war. In jene Zeit fällt auch die Anlegung des Görlitzer Berggartens, dessen Ursprünge jedoch noch in die Kriegszeit zurückreichen und interessanterweise ebenfalls auf Kraft zurückgehen.

\section{Werdegang des Gartenbaudirektors Henry Kraft}

Bereits Henry Krafts Vater, Arndt Kraft
(1868-1944), war ein geschätzter Gartengestalter. Er absolvierte eine Lehre an der Gartenbauschule in Dresden und unternahm Studienreisen durch Belgien und Holland, um danach als Gehilfe unter anderem in Breslau (Wrocław), Kassel und Windsor zu arbeiten. 1897 erfolgte seine Berufung ins schlesische Bad Salzbrunn (SzczawnoZdrój), wo er als Leiter und Garteninspektor für den Ausbau und die Betreuung der Kurparkanlagen zuständig war. Nach einer fast 30-jährigen Tätigkeit ernannte man ihn dort 1924 endlich zum »Fürstlich Plessischen Gartendirektor «. ${ }^{2} 1938$ heißt es in einer Würdigung anlässlich seines 70. Geburtstags:

»[...] Mit viel Verständnis und außerordentlichem Weitblick hat Herr Kraft es verstanden, einen Kurpark zu schaffen, um den Bad Salzbrunn von manchem Bade beneidet wird. [...] mit seinem vielseitigen Baumbestande gleicht [die Anlage] einem großen botanischen Garten. $[\ldots] \ll^{3}$

Schon zu Beginn seiner Dienstzeit in der niederschlesischen Kurstadt wurde dort am 6. April 1899 dessen Sohn Henry geboren. Dieser wuchs somit im unmittelbaren "gärtnerischen Umfeld « des Vaters auf, wo er schließlich nach Abschluss der Oberrealschule und Militärdienst im Ersten Weltkrieg von 1920 bis 1921 in die Lehre ging (Fürstlich-Pleßsche Gärtnerei). Es folgten daraufhin mehrere Jahre als Gehilfe an verschiedenen Stätten: 1921/22 Baumschule Peter in Trebnitz (Trzebnica), 1922/23 Gärtnerei Schloss Kamenz (Kamieniec 
Ząbkowicki), 1923 Handelsgärtnerei Sperlich in Gleiwitz (Gliwice) und der Schlossgarten in Waldenburg (Wałbrzych). Ab 1923 besuchte Henry Kraft die Höhere Gärtnerlehranstalt in Berlin-Dahlem, wo er 1925 als Gartenbau-Techniker abschloss. Er arbeitete anschließend bis 1927 beim Gartenarchitekten Hans Klein in Königsberg in Ostpreußen (Kaliningrad). Noch im selben Jahr beteiligte er sich an der Deutschen Gartenbauund Schlesischen Gewerbeausstellung GUGALI in Liegnitz (Legnica). ${ }^{4}$

In der Folgezeit arbeitete Kraft als freischaffender Gartenarchitekt und qualifizierte sich 1928 zum Diplom-Gartenbauinspektor. 1930 ging er in den Dienst der Stadt Liegnitz, wo er acht Jahre lang als Friedhofsinspektor tätig war, bis er sich schließlich für das Amt des städtischen Gartenbaudirektors in Görlitz bewarb und dort am 1. Januar 1939 seinen Dienst aufnahm. ${ }^{5}$ Hier trat er die Nachfolge von Oskar Sperling (18381912) und Heinrich Diekmann (1877-1941) an, die in ihrer langjährigen Tätigkeit maßgeblich zur Entstehung des Görlitzer Stadtgrüns beigetragen hatten. ${ }^{6}$ Den zeitlichen Umständen geschuldet, kam Kraft in seinen ersten Görlitzer Dienstjahren als Gestalter nicht zum Zuge. ${ }^{7}$ Erst mit dem Wiederaufbau der beschädigten und brachliegenden Grünanlagen nach dem Zweiten Weltkrieg gelang es Kraft, gleich seinen Vorgängern, seine eigene Handschrift im Stadtbild zu hinterlassen. Die Notwendigkeit wurde sozusagen zur Chance.

Nach anfänglichen Schwierigkeiten, die ihm seine vormalige Mitgliedschaft in der NSDAP bereitete, fand Kraft 1950 Anstellung bei der städtischen Park- und Friedhofsverwaltung, deren Leitung er bald übernahm. Es stellte sich nun die Aufgabe, die verbliebenen Anlagen instand zu setzen, zu erweitern bzw. auch neuen Nutzungsanforderungen anzupassen. ${ }^{8}$ Ein wichtiges Instrument zur Realisierung seiner Planungen stellte dabei das sogenannte Nationale Aufbauwerk (NAW) dar, in dessen Rahmen Werktätige, Schüler und Rentner bei unentgeltlichen (mehr oder minder freiwilligen)
Arbeitseinsätzen am Feierabend oder Wochenende Arbeitsstunden ableisteten. So wurde ein Großteil der von Kraft in den 1950er und 1960er Jahren angefertigten Entwürfe unter Mitwirkung zahlloser Görlitzer Bürger/innen ausgeführt - so beispielsweise der Nikolaizwinger (1953/54) und die Ochsenbastei (1962/63) mit ihren barockisierenden Beeten, die Freilichtbühne im Stadtpark (1955/56), das Birkenwäldchen (19551960), die Friedenshöhe (1952 bis Anfang 1960er) oder auch der Berggarten (1960/61). Für seinen unermüdlichen Einsatz beim NAW wurde Kraft unter anderem mit der Verdienstmedaille der DDR ausgezeichnet. ${ }^{9}$ Bis zu seinem Ruhestand im Jahr 1967 hatte er zahllose Neu- und Umgestaltungen von städtischen Grünflächen vorgenommen.

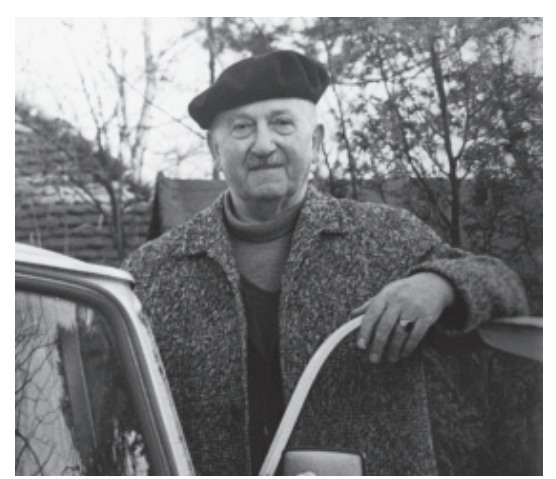

1 | Henry Kraft im Rentenalter, um 1975 (Stadtverwaltung Görlitz, Archiv SG Stadtgrün).

Die so entstandenen Gartenanlagen zeichneten sich durch farbenfrohe Blumenpflanzungen, hochwertige Natursteinarbeiten und anspruchsvolle Gehölzverwendung aus, ${ }^{10}$ was nicht nur Sachverstand, sondern auch Improvisations- und Organisationstalent erforderte. Es war Kraft gelungen, Görlitz als Gartenstadt zu einer neuen Blüte zu führen. Auch im Rentenalter stand er der Parkverwaltung noch beratend zur Seite (Abb. 1) ${ }^{11} 1979$ starb Henry Kraft im Alter von 80 Jahren. Viele seiner Planungen prägen bis heute das Grün der Neißestadt. 


\section{Entstehung des Berggartens im Zweiten Weltkrieg}

Der am Rand der Görlitzer Südstadt gelegene Berggarten geht ursprünglich auf einen privaten Garten zurück. In den 1920er Jahren entstand, zwischen Zittauer Straße und Landskronbrauerei gelegen, ein neues Villenviertel. 1923 ließ der Fabrikbesitzer Richard Rau an der südwestlichen Grenze dieser Siedlung, in der Heinzelstraße 9, ein landhausartiges Wohngebäude errichten, wofür der Görlitzer Architekt Alfred Hentschel die Pläne lieferte. ${ }^{12}$ Ein hohes Mansarddach mit allseits herausragenden Gauben sowie ein rustikal mit Granitsteinen verkleidetes Souterrain zeichnen das Bauwerk aus. Besonders markant ist ein auf der Südseite hervortretender Altan (Abb. 2). Unterhalb davon befindet sich eine Terrasse, von der aus eine seitliche Freitreppe in den Garten führt. Die erhöhte Lage des Hauses ermöglicht weite Blicke über die Weinberge ins Neißetal, bis zum Isergebirge und zur Landeskrone.

Vermutlich Mitte oder Ende der 1930er Jahre erwarb der Kaufmann Kurt Winkler, Inhaber eines Fotogeschäfts, das Haus samt dem dazugehörigen, $2.750 \mathrm{~m}^{2}$ großen Grundstück. 1940 kaufte er eine südlich angrenzende, mit $9.100 \mathrm{~m}^{2}$ wesentlich größere Freifläche hinzu, um den bestehenden Villengarten zu erweitern. Es handelte sich dabei um einen ehemaligen Weinberg. Für die Gestaltung des Geländes engagierte Winkler den befreundeten Gartenarchitekten Henry Kraft. ${ }^{13}$

Die Arbeiten am Winklerschen Garten begannen 1941 und setzten sich bis ca. 1943 fort. ${ }^{14}$ Angesichts der Tatsache, dass sich Deutschland mitten im Krieg befand und an den Fronten abertausende Soldaten fielen, erscheint dies zunächst etwas paradox. ${ }^{15}$ Kraft, der die Ausführung leitete (Abb. 3), war zu jener Zeit vom Kriegsdienst zurückgestellt und für die Ernährungswirtschaft tätig. ${ }^{16} \mathrm{Da}$ die meisten Männer damals zum Militär eingezogen wurden, führten mehrere ältere Herren sowie zwei junge, vermut- lich beurlaubte Wehrmachtsoldaten die umfangreichen Pflanzarbeiten durch (Abb. 4). Es ist bemerkenswert, wie es Kraft gelang, in Zeiten des allgemeinen Mangels und Notstands die beachtlichen Mengen an Gehölzen (ca. 200 Bäume und Sträucher) zu beschaffen (Abb. 5).

Als Ausgangssituation für die Anlage bot sich eine weitläufige Wiesenfläche mit wenigen, wild gewachsenen Bäumen an - darunter auch eine malerische Birkengruppe im Zentrum, die beibehalten und in die Gartengestaltung einbezogen wurde. ${ }^{17}$ Für die hinzukommenden Neupflanzungen wählte Kraft vorzugsweise verschiedene Wacholder-, Kiefern- und Rhododendron-Arten sowie wiederum Birken, weshalb die Vermutung nahe liegt, dass er vorhatte, einen Heidegarten anzulegen. Da sich allerdings die Verwendung von Heidekraut als essentieller Bestandteil und auch das typische Findlingsmotiv nicht nachweisen lassen, bleibt offen, ob es diese gestalterische Intention je gab. Fakt ist, dass immergrüne Gehölze, insbesondere Koniferen zum Hauptthema bestimmt wurden. ${ }^{18}$

Auch wenn die wichtigsten Pflanzarbeiten damals offenbar zum Abschluss kamen, kann man davon ausgehen, dass der Winklersche Garten vor Kriegsende nicht komplett fertig gestellt wurde. ${ }^{19}$

\section{Umwandlung zur öffentlichen Grünan- lage in der DDR}

1960 sollte der brachliegende Winklersche Garten in eine öffentliche Grünanlage umgewandelt werden. Winkler selbst hatte die DDR inzwischen aus politischen Gründen verlassen und sein Grundstück stand jetzt unter staatlicher Verwaltung. Das weiterhin für Wohnzwecke genutzte Haus wurde nunmehr vom eigentlichen Garten abgetrennt - eine Situation, die bis heute besteht (Abb. 6). ${ }^{20}$

Kraft nahm sich jetzt ein zweites Mal der Anlage an, diesmal jedoch in der Funktion als städtischer Gartenbaudirektor. Es galt, den vormals privaten Garten einer öffentli- 


\section{Henry Kraft und der Görlitzer Berggarten}

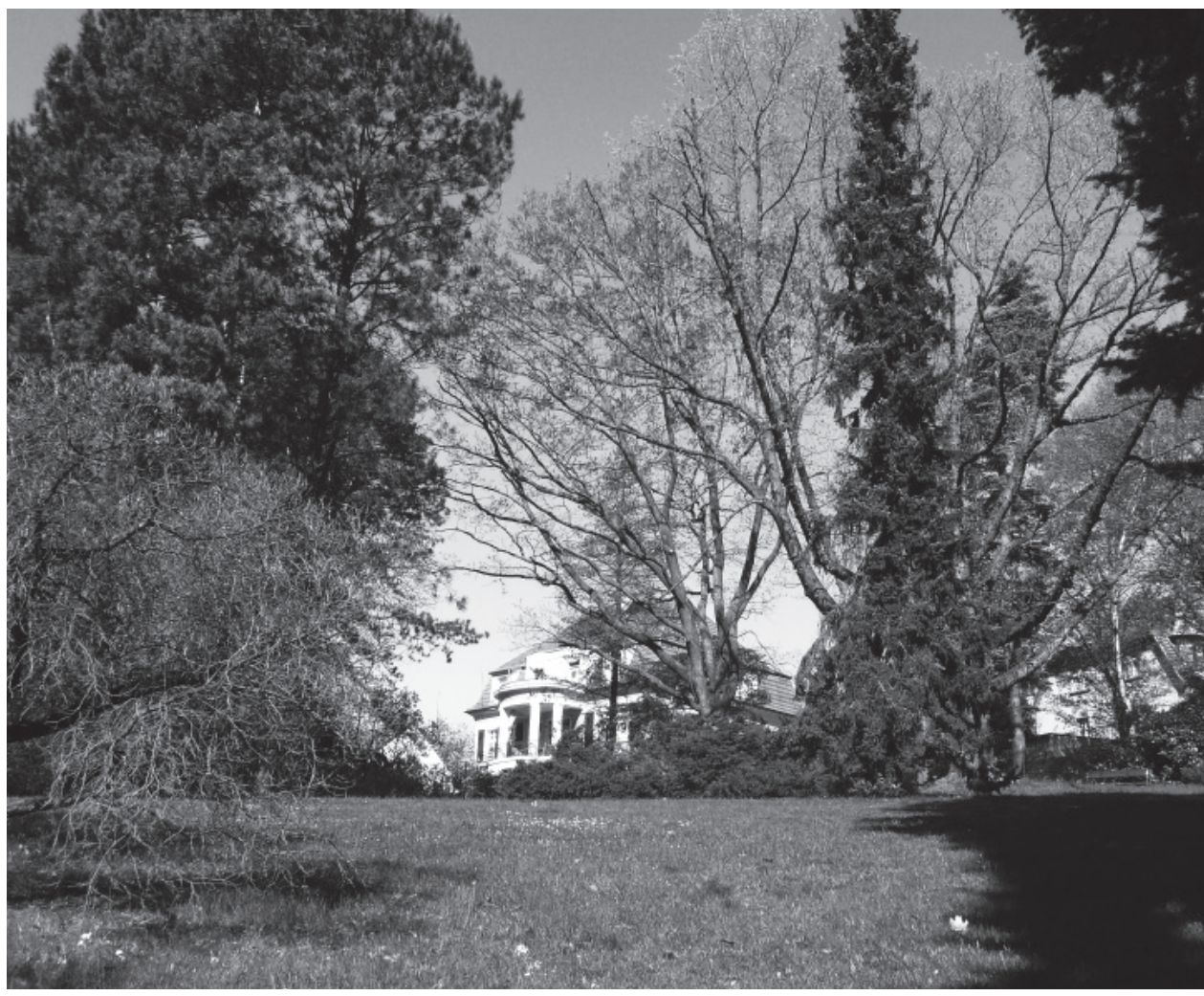

2 | Blick durch den Berggarten zur Villa Heinzelstraße 9 im Norden, Fotografie, Christoph Haase 2014.

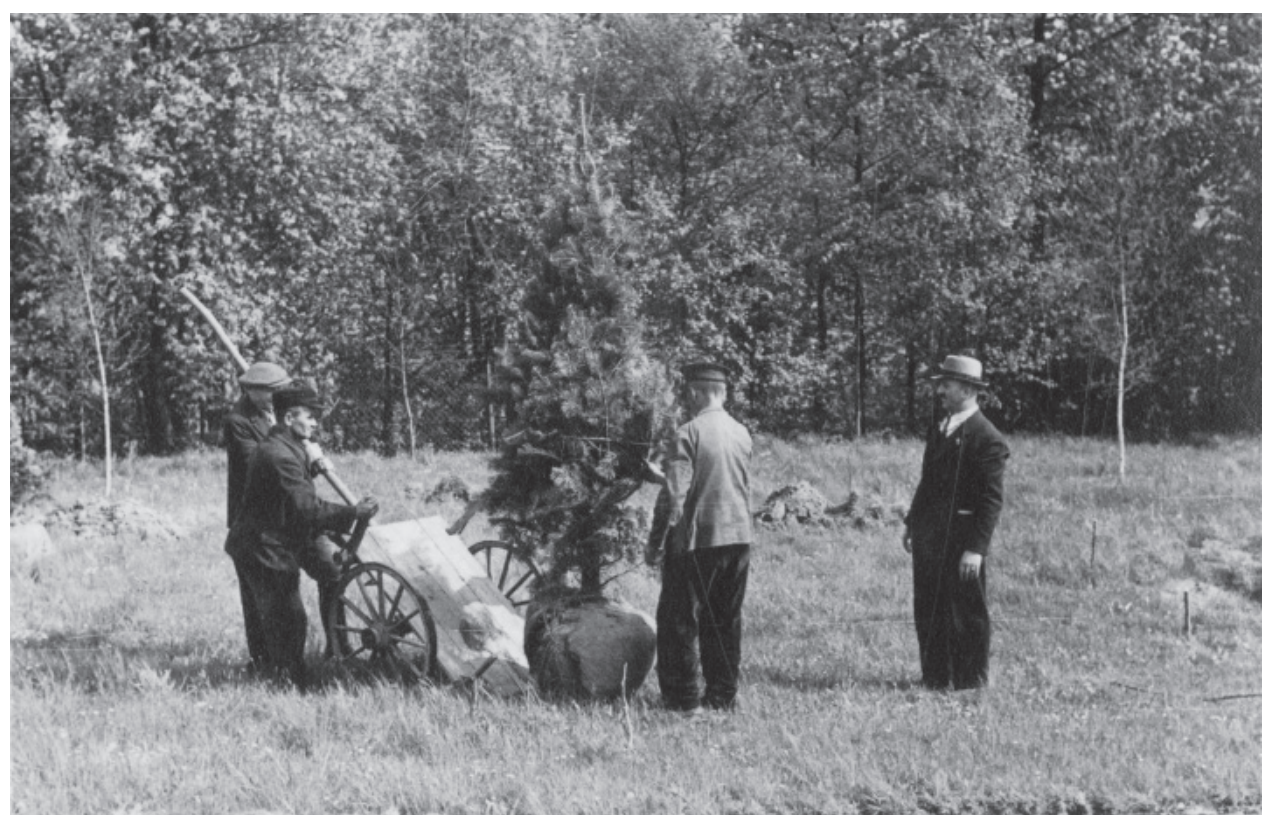

3 | Positionieren einer Schwarz-Kiefer im Winklerschen Garten unter Anleitung von Henry Kraft (rechts), Fotografie, vermutlich Kurt Winkler 1943 (Ratsarchiv Görlitz). 


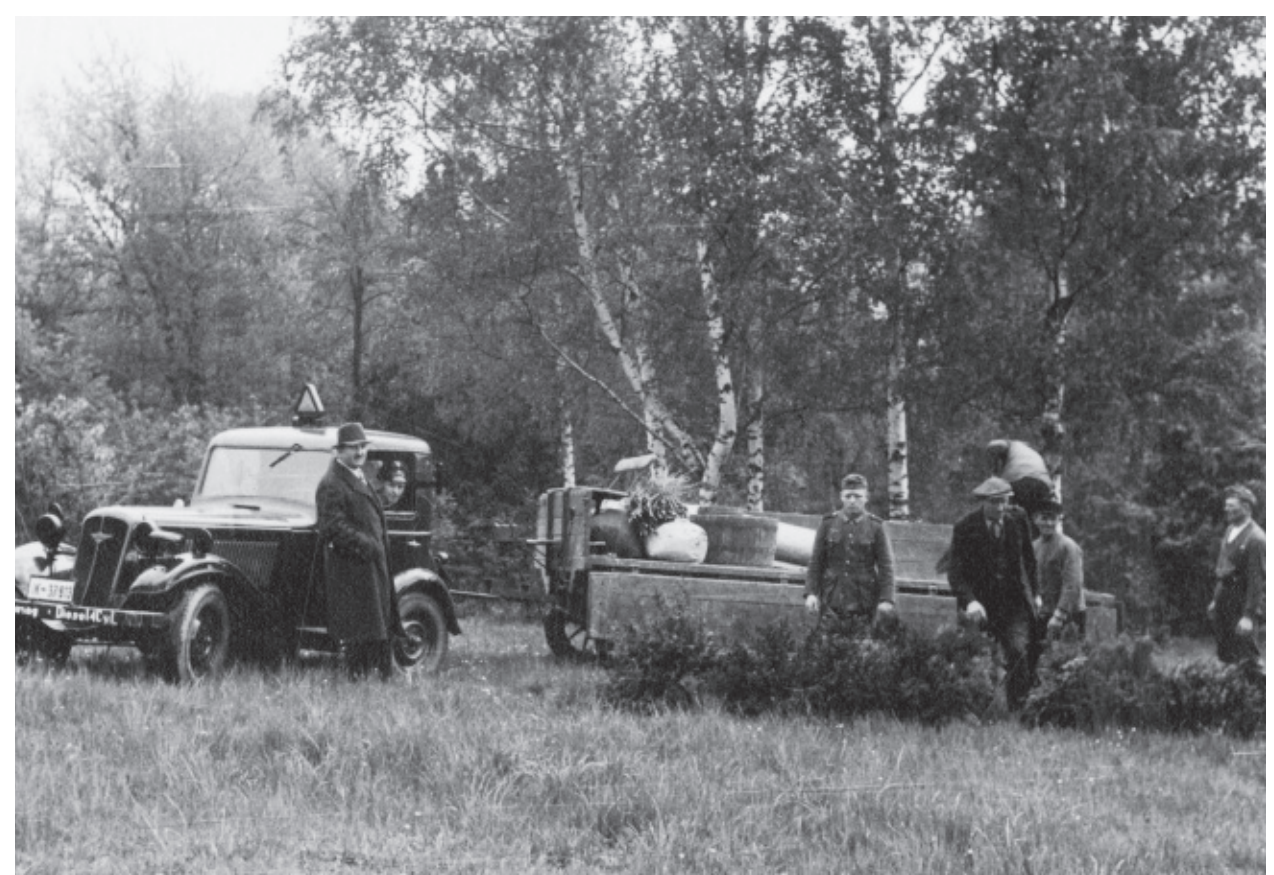

4 | Soldaten und Hilfsarbeiter beim Entladen einer Gehölzlieferung nahe der zentralen Birkengruppe, ganz links Henry Kraft, Fotografie, vermutlich Kurt Winkler 1943 (Ratsarchiv Görlitz).

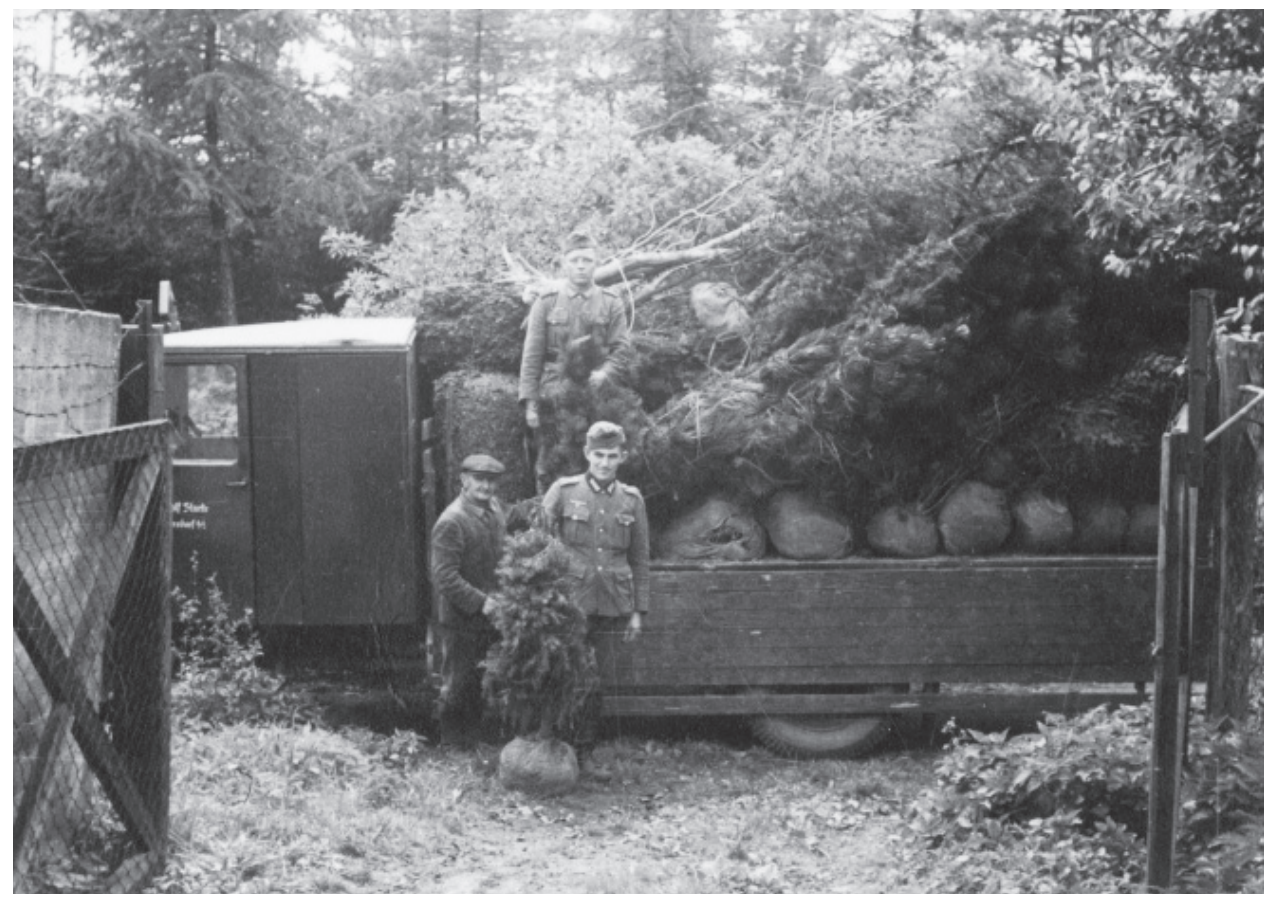

5 | Gehölzlieferung für den Winklerschen Garten mit Bäumen und Sträuchern in teils beachtlich großer Qualität, Fotografie, vermutlich Kurt Winkler 1943 (Ratsarchiv Görlitz). 


\section{Henry Kraft und der Görlitzer Berggarten}

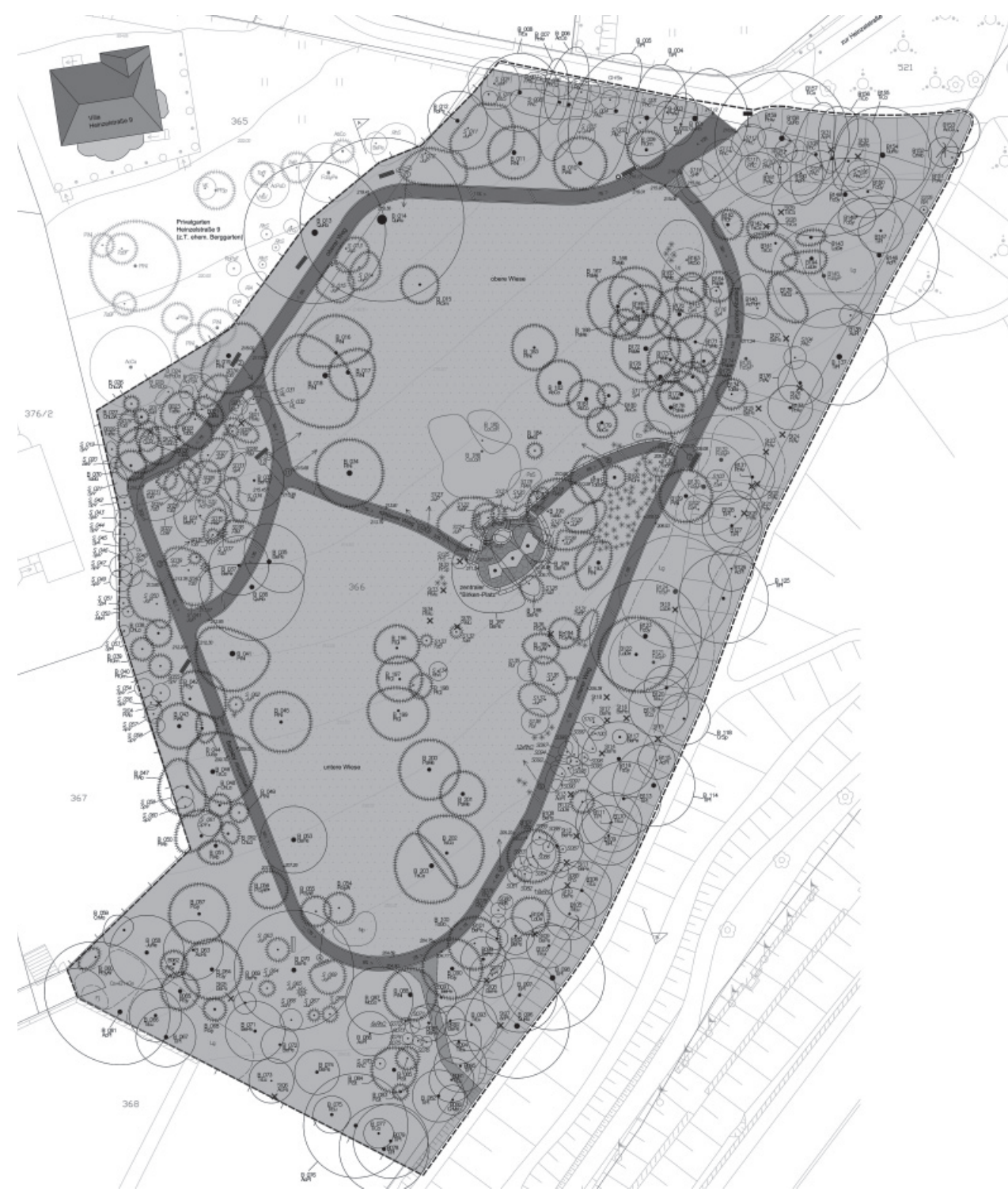

6 | Bestandsplan des Berggartens, oben links die ehemals dazugehörige Villa, CAD, Christoph Haase 2014.

chen Nutzung anzupassen. Dementsprechend entstanden ein fest ausgebautes Wegesystem und verschiedene Sitzplätze. Um das malerische Birkenensemble im Zentrum des Gartens legte Kraft einen intensiv gestalteten Platz an, der terrassenartig aus dem Hang ragt. Trockenmauern aus bruchrauen Granitblöcken stützen die aus polygonalen Schieferplatten bestehende Fläche ab. Unterhalb und seitlich davon gab es Stauden- pflanzungen (Abb. 7). Der Gehölzbestand erhielt durch die Neupflanzung von auffälligen Ziergehölzen (zum Beispiel TulpenMagnolien, Fächer-Ahorne) und besonderen Solitärs (zum Beispiel Trauer-Birke und Urweltmammutbaum) ein frisches Aussehen. Auch hatte Kraft manche Rarität im Berggarten verwendet, wie Amerikanischer Gagelstrauch (Myrica pensylvanica) und Glockige Prachtglocke (Enkianthus campa- 
nulatus), was die Anlage auch aus dendrologischer Sicht interessant erscheinen ließ. ${ }^{21}$

Am 1. Mai 1961 wurde die in zahlreichen Arbeitseinsätzen des NAW errichtete Anlage feierlich unter dem Namen »Berggarten « eröffnet. Die Stadt war nun um eine bedeutende Grünanlage reicher (Abb. 8-10).

\section{Gestaltung und Erscheinungsbild}

Der nur etwa einen Hektar große, landschaftlich gestaltete Berggarten bildet den südwestlichen Abschluss eines großen Grünzugs, der sich vom Neißeviadukt bis zur Zittauer Straße entlang der Neißehänge erstreckt. Wer sich dem Berggarten von der Innenstadt her nähert, passiert die Parkanlagen Friedenshöhe und Schellergrund, durchquert die schattigen Weinberganlagen und kommt am Freizeitareal der Parkeisenbahn sowie der Heinzelwiese vorbei. Der Gebäudekomplex der Landskronbrauerei sowie das historische Weinberghaus mit dem benachbarten Aussichtsturm sind in dieses grüne Band eingebettet. Als letztes Glied in der Kette aus historischen Parks,
Gärten und Bauwerken folgt der Berggarten mit einer eher abgeschiedenen Lage. Hier herrscht eine intime Atmosphäre. Relief, räumliche Anordnung und Pflanzenauswahl sorgen für ein abwechslungsreiches Erscheinungsbild, das im Wechsel der Tages- und Jahreszeiten stets neue Reize hervorbringt.

Die prägnante Hanglage ist die Grundlage für die spannungsvolle Gestaltung und bedingt interessante Blickführungen - unter anderem zu den angrenzenden Villen (Abb. 2, 11). Bereits am Eingang öffnet sich eine eindrucksvolle Sicht in den Garten, jedoch ohne dass dieser schon in Gänze überschaut werden kann. Die Geländeoberfläche fällt relativ gleichmäßig nach Süden bzw. Südosten ab (Abb. 12). Es lassen sich prinzipiell drei gestalterische Höhenebenen unterteilen, die unmerklich ineinander übergehen. Der höher gelegene, nördliche Teil ist weiträumig und lichtdurchflutet. In der mittleren Ebene bewirken vielgestaltige Gehölzgruppen unterschiedliche Lichtverhältnisse. Die unteren Partien im Süden und Südosten sind durch den waldartigen Baumbestand ganztägig kühler und dunkler. Dementspre-

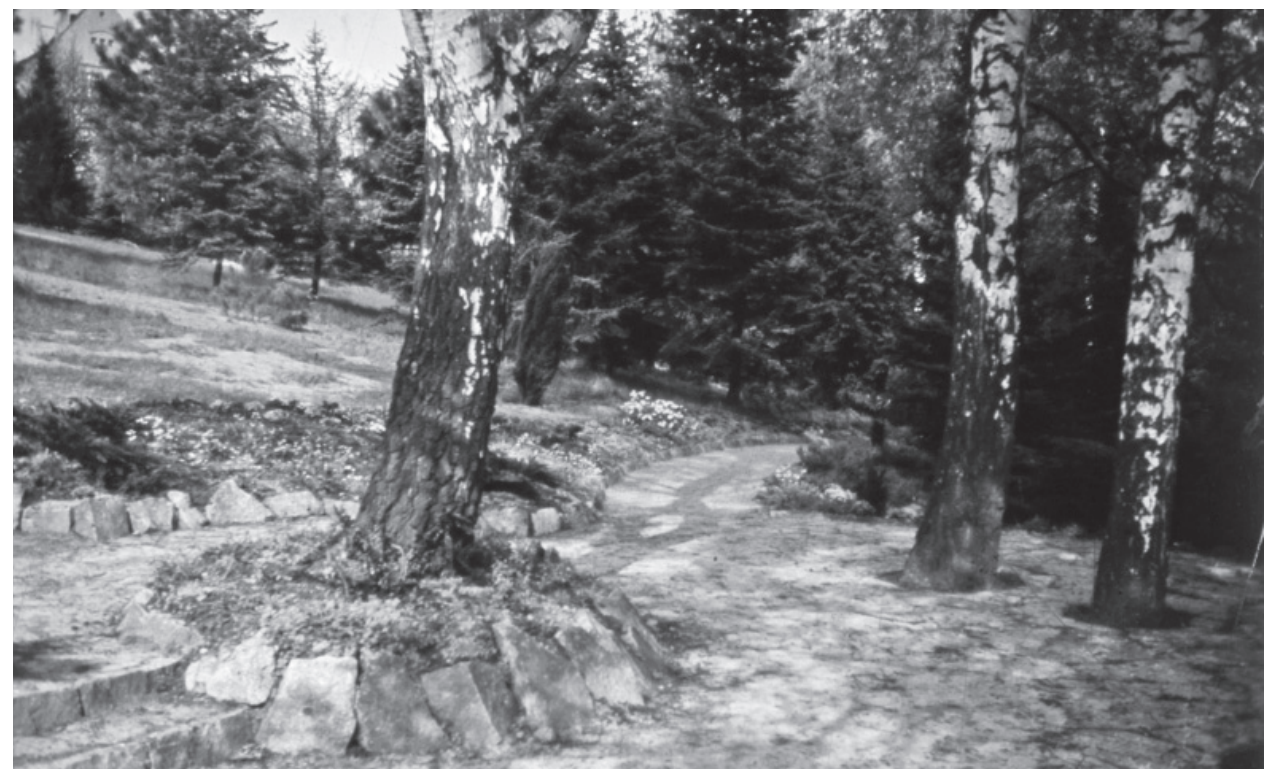

7 | Zentrale Platzfläche mit der markanten Birkengruppe kurz nach der Fertigstellung, Fotografie, Henry Kraft, Frühjahr 1961 (Ratsarchiv Görlitz). 
chend findet man verschiede Verweilmöglichkeiten in sonniger bis tiefschattiger Lage. Ein schlichtes und zweckmäßiges Wegesystem erschließt die einzelnen Bereiche des Gartens. Es passt sich harmonisch an Grundriss und Geländeform der Anlage an (Abb. 6). Zentrales Element der Gesamtgestaltung ist die kleine Platzfläche aus Naturstein mit der bereits erwähnten markanten Birken-Gruppe. ${ }^{22}$ Die dorthin führenden Verbindungswege durchschneiden die von einem großen Rundweg eingeschlossene Wiesenfläche.

Die dichte Bepflanzung der Randbereiche schirmt den Gartenraum nach außen ab und fungiert zugleich als Hintergrundkulisse für die innere Gestaltung. Diesem größtenteils geschlossenen Gehölzgürtel sind Solitärbäume und vielfältige Baumgruppen vorgelagert, die weit in den Wiesenraum übergreifen. Während an den Rändern Laubund Nadelbäume gemischt vorkommen, dominieren im Innern Koniferen. Bäume und Sträucher sind zudem hinsichtlich Farbe und Habitus äußerst kontrastreich gewählt und kombiniert. So stehen beispielsweise
Birken mit ihrer weißen Rinde, dem hellgrünen Laub und locker herabhängenden Zweigen im klaren Gegensatz zu dunklen, kegelförmigen, eher statisch wirkenden Fichten (Abb. 11). Flach ausgebreitete Wacholder betonen die Horizontale, säulenförmige Eiben und Fichten die Senkrechte. Als Solitäre stechen vor allem einige malerische Schwarz-Kiefern sowie eine beeindruckende Rot-Eiche mit kandelaberartigem Wuchs hervor. Rotblättrige Gehölze wie Blut-Buchen setzen farbliche Akzente im Bestand.

Nicht zuletzt sorgen die jahreszeitlichen Aspekte für farbliche Abwechslung. Im Winter bilden die hellen Stämme der Birken und das dunkle Laub der Immergrünen ein gewisses Grundgerüst. Vom Frühjahr bis in den Sommer hinein blühen unterschiedliche Gehölze in reicher Fülle: Zier-Apfel, Magnolien, Azaleen, Rhododendren, Spiersträucher und andere. Im Herbst verwandeln die Laubbäume den Park in ein buntes Farbenmeer. Besonders attraktiv präsentiert sich dann eine außergewöhnlich große und alte Gruppe von Perückensträuchern in der Mitte des Gartens.

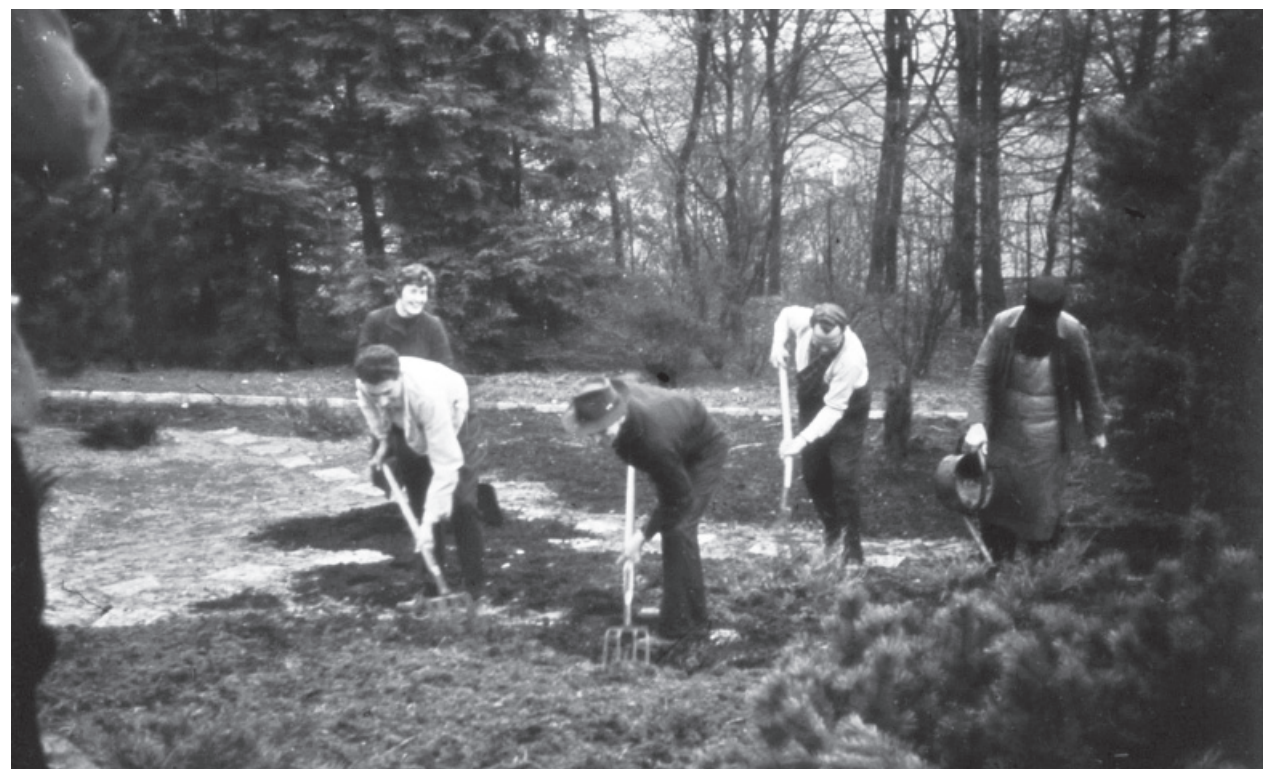

8 | Herstellen einer Pflanzfläche unterhalb des zentralen Platzes durch Helfer des Nationalen Aufbauwerks, Fotografie, Henry Kraft, Winter 1960/1961 (Ratsarchiv Görlitz). 


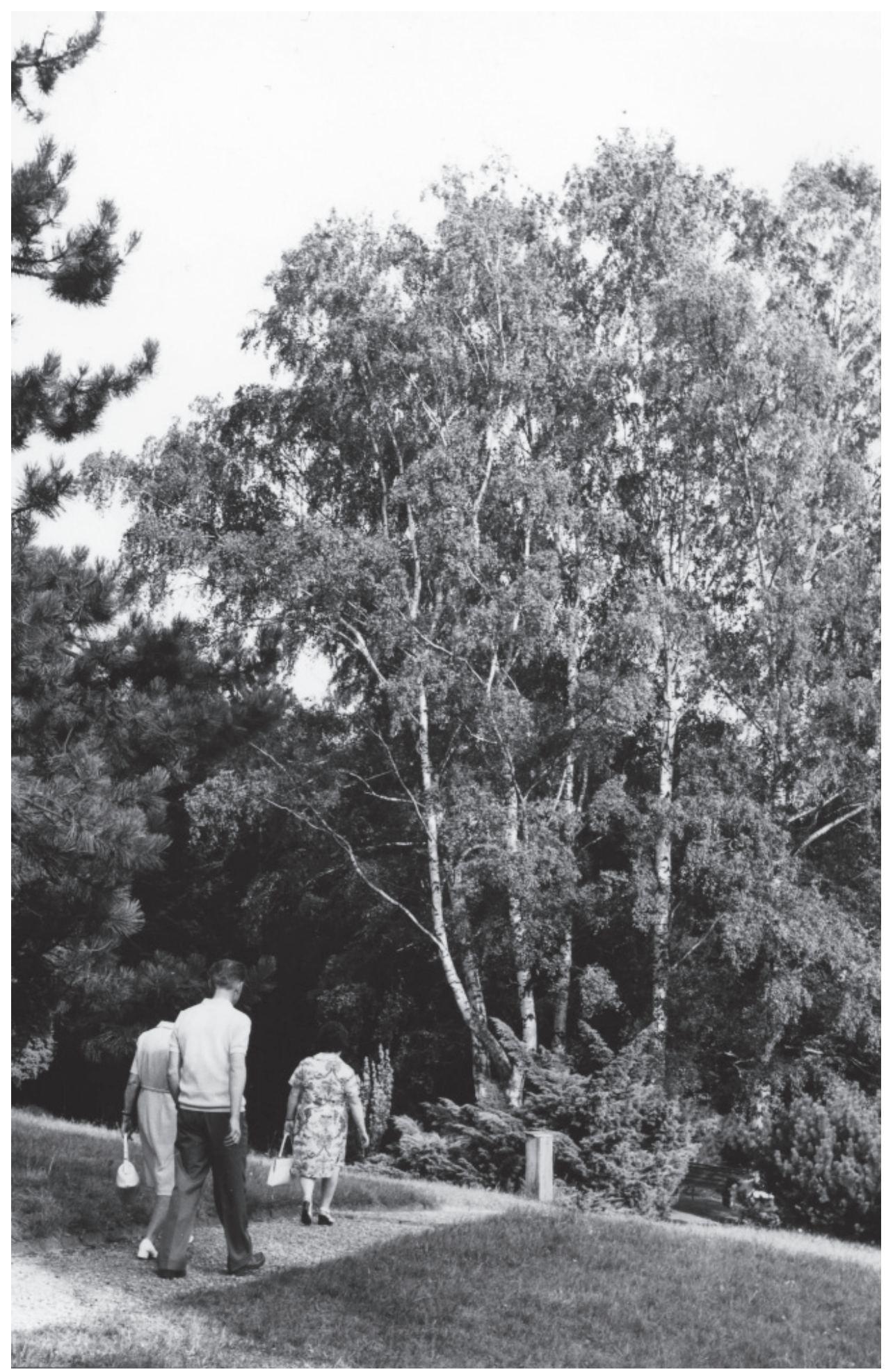

9 | Blick vom westlichen Gartenteil auf den zentralen Platz mit der Birkengruppe, Fotografie, Heinz Pfennig, Ende 1970er Jahre (Stadtverwaltung Görlitz, Archiv SG Stadtgrün). 


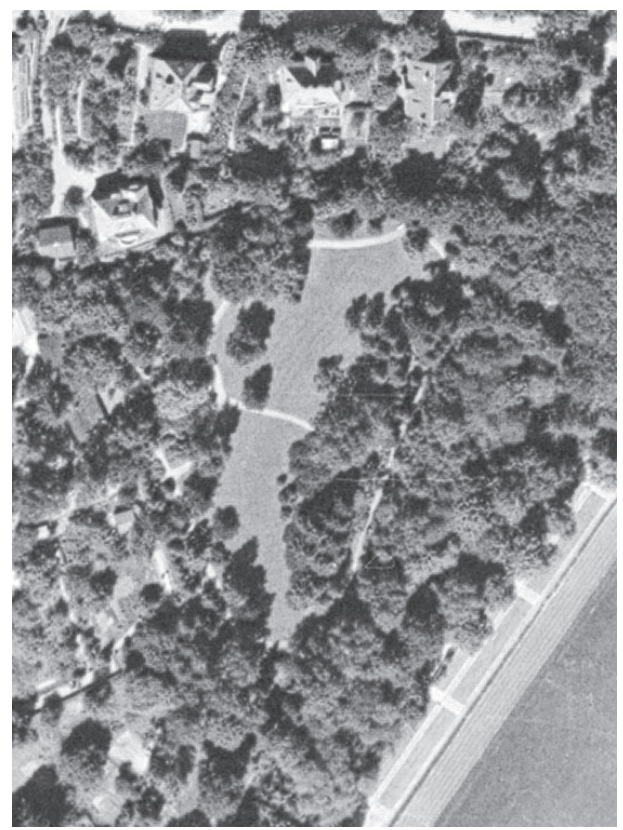

10 Der Berggarten mit den nördlich angrenzenden Villen der Heinzelstraße auf einem Luftbild von 1973 (BArch, Bild 180, Film-Nr. C253695/73, Bild-Nr. 388).

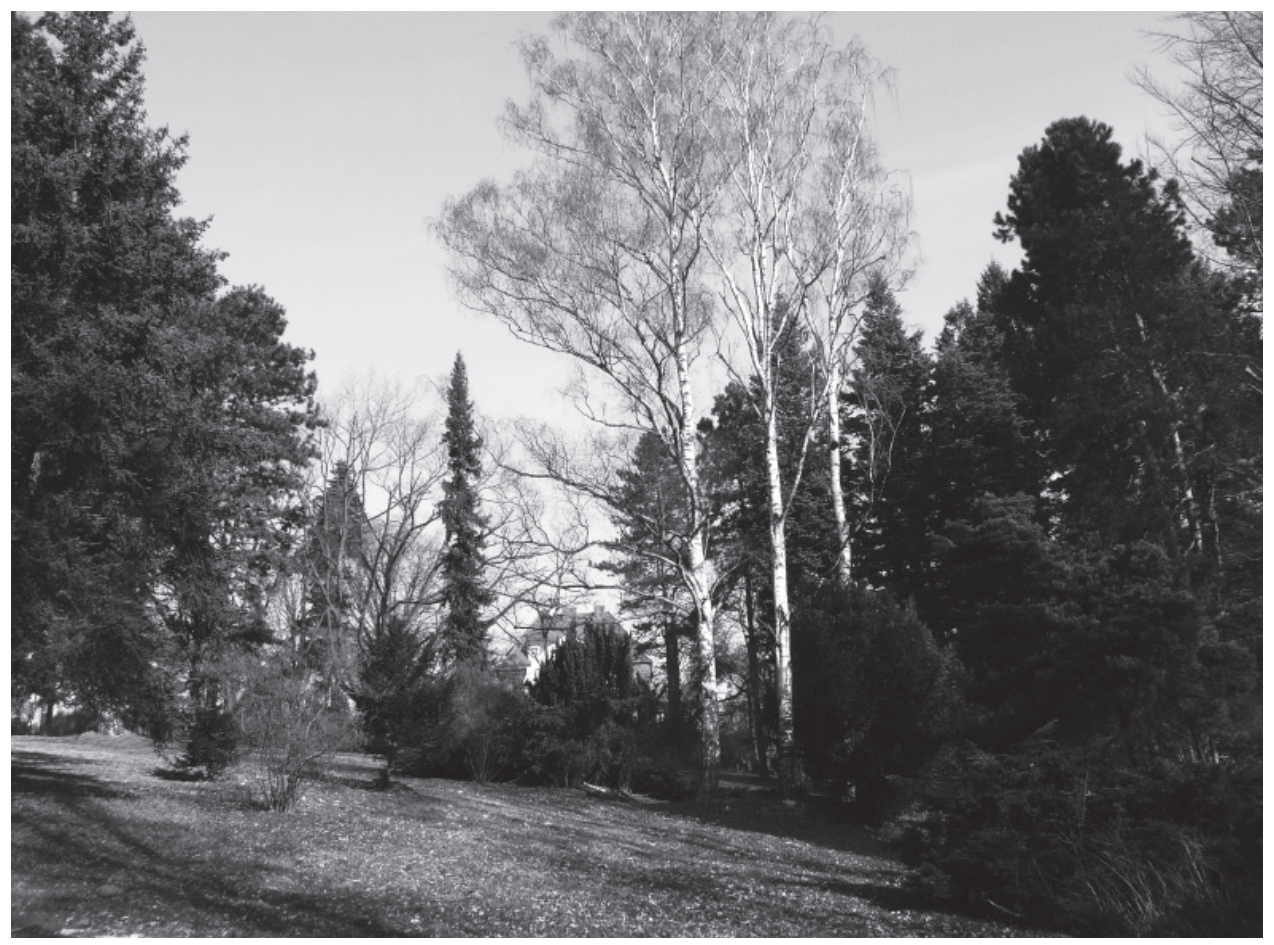

11 | Blick vom unteren Weg nach Norden. In der rechten Bildhälfte sind drei der ursprünglich fünf Birken des zentralen Platzes, im Hintergrund die Villen Heinzelstraße 7 und 6 zu sehen, Fotografie, Christoph Haase 2014. 


\section{Der Berggarten als Gartendenkmal heute}

Erst in den frühen 1940er Jahren entstanden, ist der Berggarten Zeugnis einer späten, bürgerlichen Villengartenkultur in der ersten Hälfte des 20. Jahrhunderts. Als großzügig und landschaftlich gestaltete Anlage hebt er sich im Vergleich zu anderen, meist geometrischen und kleineren Villengärten der Görlitzer Südstadt deutlich hervor. Durch seine Umgestaltung zur öffentlichen Grünanlage im Rahmen des NAW der DDR ist er ebenso Zeugnis sozialistischer Aufbauarbeit nach dem Zweiten Weltkrieg. In ihm spiegelt sich somit der gesellschaftliche und politisch-ideologische Wandel jener Zeit wider. Nicht zuletzt ist er ein Werk des Görlitzer Gartenbaudirektors Henry Kraft, dessen Planungen beispielhaft für die vielfältige Freiraumgestaltung der 1950er und 1960er Jahre in der DDR stehen können. ${ }^{23}$

Noch heute bietet die Anlage eine hohe Aufenthaltsqualität und zeichnet sich nach wie vor durch einen bemerkenswerten $\mathrm{Ge}$ hölzbestand aus. Letzterer ist jedoch aus gartendenkmalpflegerischer Sicht eine Herausforderung. Häufig sind die lichtbedürftigen Koniferen und Birken von den mittlerweile hoch gewachsenen Buchen und Linden bedrängt. Einige Bäume sind bereits durch Platz- bzw. Lichtmangel abgängig. Die veränderten Bedingungen erschweren dem- entsprechend auch Nachpflanzungen. Hinzu kommt, dass einige Gehölze (vor allem Wacholder) durch Überalterung nach und nach ausfallen. Wo es die Standortverhältnisse ermöglichen, lassen sich abgestorbene Pflanzen ersetzen. In manchen Partien wird man allerdings den natürlichen Wandel und den damit einhergehenden Verlust einiger, teils charakteristischer Arten zugunsten etablierter Altbäume langfristig hinnehmen müssen.

Großer Handlungsbedarf besteht, trotz großer Bemühungen seitens der Stadt, vor allem bei den Sitzplätzen sowie den wassergebundenen Wegen. Überhaupt gestaltet sich die Pflege der Anlage angesichts knapper Mittel schwierig, wenn man bedenkt, dass sie nur einen Bruchteil der weitläufigen Grünflächen von Görlitz darstellt. Hinzu kommt die abgeschiedene Lage, mit der vermutlich auch die geringe Nutzerzahl einhergeht. Abhilfe könnte hier eine stärkere Einbindung in das touristische Informationssystem unter Berücksichtigung des gesamten Grünzugs bringen, zumal Anziehungspunkte wie die Brauerei, Parkeisenbahn oder das auf Wiederbelebung wartende Weinberghaus nicht weit entfernt sind. Auch die Görlitzer/innen selbst sind gefragt, »ihren « Berggarten wiederzuentdecken warum nicht bei einem gemeinsamen Arbeitseinsatz in Form eines Parkseminars?

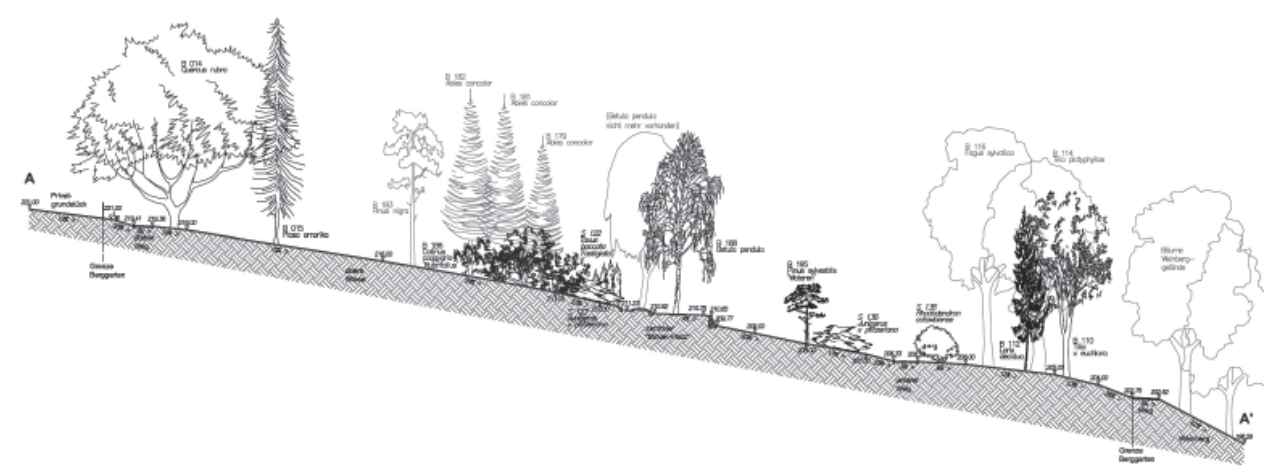

12 | Geländeschnitt des Berggartens, Blick nach Osten, CAD, Christoph Haase 2014. 


\section{Henry Kraft und der Görlitzer Berggarten}

1 Zur Entwicklungsgeschichte des Görlitzer Stadtgrüns s. u. a. Stadtverwaltung Görlitz/Amt 66/ Sachgebiet Stadtgrün: Entwicklung der Görlitzer Parkanlagen. Geschichte der Görlitzer Parkverwaltung, Görlitz (Stand: 05.05.2014) [unveröffentlichtes Manuskript].

2 Gröning, Gert; Wolschke-Bulmahn, Joachim: Grüne Biographien. Biographisches Handbuch zur Landschaftsarchitektur des 20. Jahrhunderts in Deutschland, Berlin/Hannover 1997, S. 200; 1924 heißt es: "Herr Krafft [sic] ist seit fast 20 [gemeint sind 30] Jahren in den Fürstl. Plessischen Diensten und erfreut sich großen Ansehens und allgemeiner Beliebtheit.« (Anonymus: Persönliche Nachrichten - Krafft, Arndt, in: Die Gartenwelt H. 45/1924, S. 536).

3 Jeschke: Gartendirektor i.R. Arndt Kraft, in: Die Gartenkunst H. 4/1938, S. 4.

4 Vgl. Höhere Gärtnerlehranstalt Berlin-Dahlem [Hg.]: Denkschrift zum 100jährigen Bestehen der höheren Gärtnerlehranstalt Berlin-Dahlem - früher Wildpark, Frankfurt (Oder) 1924, S. 216 sowie Hill, Thomas: Leben und Wirken des Görlitzer Gartenbaudirektors Henry Kraft (1899-1979), Dresden 1999, S. 2 [unveröffentlichte Semesterarbeit, TU Dresden].

5 Vgl. Wetter, Christian: Persönliche Nachrichten Henry Kraft, in: Die Gartenwelt H. 48/1930, S. 672 und Hill, S. 2 f.

$6 \mathrm{Zu}$ Sperling s. Anonymus: Personalnachrichten Sperling, F. [sic], in: Die Gartenwelt, H. 51/1904, S. 607; zu Diekmann s. Freudrich, Christian: Der Kreuzkirchenpark in Görlitz, in: Denkmalpflege in Sachsen. Mitteilungen des Landesamtes für Denkmalpflege Sachsen, Dresden 2016, S. 105-113, hier S. $108 f$.

7 In den Zeitraum 1939-1945 fallen keine bemerkenswerten Gestaltungen, lediglich einige Veränderungen, wie z.B. am Postplatz, Wilhelmsplatz oder im Bereich des Parkhauses im Stadtpark (Hill 1999, S. 10).

8 Ebd., S. 3f., 10f. Der östlich der Neiße gelegene Teil von Görlitz ging nach 1945 an Polen. Dementsprechend gab es Bedarf an öffentlichem Grün.

9 Vgl. ebd., S. 9f.; Sander 1980, S. 19-24; Stadtverwaltung Görlitz/Amt 66/Sachgebiet Stadtgrün 2014.

10 Vgl. Diasammlung Henry Krafts im Ratsarchiv Görlitz (RAG).

11 Hill 1999, S. 5.

12 Frank-Ernest Nitzsche: Denkmalpflegerische Zielstellung für das Landhaus Heinzelstraße 9 in Görlitz, Görlitz 1995 [unveröffentlicht, Untere Denkmalschutzbehörde Görlitz].

13 Das Jahr 1940 für den Erwerb des ehemaligen Weinberggeländes sowie das freundschaftliche Verhältnis zwischen Winkler und Kraft gehen aus einem juristischen Schreiben vom 10.9.1993 in der Akte Berggarten der Stadtverwaltung Görlitz, Amt 66/Sachgebiet Stadtgrün hervor (Stand Mai 2014).

14 Das Jahr 1941 taucht in den Quellen allgemein als
Entstehungsjahr auf. Bei Sander 1980, S. 21 werden für die Bauzeit die Jahre 1941-1942 angegeben. Die Diaaufnahmen, die die umfangreichen Pflanzarbeiten im Garten dokumentieren, sind im Gesamtverzeichnis der Diasammlung Henry Krafts (RAG) auf Juli 1943 datiert.

15 Das Infanterie-Regiment 30 Görlitz/Lauban verlor bei Gefechten insgesamt 2.800 Soldaten. 1940 fielen erste britische Fliegerbomben auf Görlitz. 1942 ließ man die meisten Bronze-Denkmäler und Glocken der Stadt zu Rüstungszwecken demontieren. Auch die öffentlichen Plätze und Gärten wurden stark in Mitleidenschaft gezogen (Hoche, Siegfried: Chronik der Stadt Görlitz, Nordhorn 2006, S. 34-36). Der Fakt, dass Görlitz bis 1945 von schwereren Angriffen verschont blieb, begünstigte sicherlich die Umstände zum Anlegen des Gartens. Vieles spricht dafür, dass der Kaufmann Winkler ein hochrangiges Mitglied der NSDAP war (und eine vom Sieg des Dritten Reiches überzeugte Person dazu).

16 Hill 1999, S. 3.

17 Vgl. Sander 1980, S. 21 und Diasammlung Henry Krafts im RAG.

18 Vor allem durch den Gartentheoretiker Willy Lange (1864-1941) zu Beginn des 20. Jahrhunderts ausgelöst, wurde das Heidemotiv bis in die 1940er Jahre von nationalistischen Ideologien vereinnahmt. Es stand für eine naturnahe und heimatliche (»deutsche«) Gartengestaltung. Vgl. Gröning, Gert; Schneider, Uwe: Die Heide in Park und Garten. Zur Geschichte und Bedeutung des Heidemotivs in der Gartenkultur, Worms 1999, S. 46-49, 116-130.

19 Die Existenz von Sitzplätzen, Staudenpflanzungen usw. bis 1945 lässt sich heute nicht mehr nachvollziehen. Auf einer Luftaufnahme vom 18.2.1945 (Luftbilddatenbank Dr. Carls GmbH Würzburg) sind lediglich die jungen Gehölzpflanzungen und ein spärliches Wegesystem erkennbar.

20 Juristisches Schreiben vom 28.4.1994 (Stadtverwaltung Görlitz, Amt 66/Sachgebiet Stadtgrün - Akte Berggarten).

21 Sander 1980, S. 13, 15.

22 Man würde an dieser Stelle eine Aussicht auf die Neißeaue erwarten, jedoch ist der Gehölzgürtel im Osten dicht geschlossen. Bereits beim Winklerschen Garten hatte es hier nachweislich (Diasammlung Henry Krafts im RAG) keine Blickverbindung gegeben, was wahrscheinlich daran lag, dass man eine Sicht auf die am Fuße der Weinberge errichteten Baracken eines Reichsarbeitsdienstlagers vermeiden wollte. Zum Ende des Krieges diente das Lager der Unterbringung von Zwangsarbeitern (für den Hinweis dankt der Autor Kai Wenzel). In der DDR entstanden an dieser Stelle das Stadion der Freundschaft sowie das Volksbad.

23 Kraft war es übrigens vergönnt, Gartenschauen in der BRD zu besuchen (Hill 1999, S. 5). In seiner Diasammlung (RAG) befinden sich bspw. Aufnahmen von der Bundesgartenschau in Köln 1957. 